\title{
Telephone follow-up in an occupational rehabilitation programme - a randomised controlled trial
}

Karen Walseth Hara ${ }^{1,2,3,4}$, Johan Håkon Bjørngaard ${ }^{1,5}$ Søren Brage ${ }^{6}$, Petter Christian Borchgrevink ${ }^{2,3}$, Vidar Halsteinli1 ${ }^{1,7}$, Tore Charles Stiles ${ }^{8}$, Roar Johnsen ${ }^{1}$, Astrid Woodhouse ${ }^{1,2,3}$

Transfer from on-site rehabilitation to the participant's daily environment is considered a weak link in the rehabilitation chain. Various follow-up regimes have been implemented after multidisciplinary rehabilitation, however, consensus is lacking on recommended content, duration and intensity.

\section{Questions \\ Main question: Does adding boosted return-to-work (RTW) follow-up by telephone after completing an occupa- tional rehabilitation programme effect work participation over the first year for individuals on long-term sick leave? Secondary question: What is the added cost of boosted follow-up from the perspective of the occupational rehabil- itation institution?}

\begin{abstract}
Methods
A randomised controlled trial included participants of working age with chronic pain, chronic fatigue or common mental disorders on long-term sick leave ( $>8$ weeks' duration). Participants completed a 31/2-week on-site occupational rehabilitation programme based on Acceptance and Commitment Therapy. Participants were block-randomised to boosted follow-up or control group before returning to their daily environments. The intervention was delivered over six months by RTW coordinators, mainly via telephone. Primary outcome was entry/re-entry to work defined as participation in competitive work $\geq 1$ day per week on average over eight weeks. Sensitivity analyses, used the outcome measures 'minimum half-time work', defined as 2.5 days ( $18.75 \mathrm{hrs}$ ) per week on average over 8 weeks, and 'full-time work', defined as $\geq 4$ days ( $30 \mathrm{hrs}$ ) per week on average over eight weeks, as more robust measures of
\end{abstract}

\footnotetext{
1,2,3 See affiliations 1, 2 and 3 on page 358

${ }^{4}$ The Norwegian Labour and Welfare Service of Trøndelag, Trondheim, Norway Forensic Department and Research Centre Brøset, St. Olavs Hospital, Trondheim University Hospital, Trondheim, Norway

The Norwegian Directorate for Labour and Welfare, Oslo, Norway

Centre for Health Care Improvement, St. Olavs Hospital, Trondheim University Hospital, Trondheim, Norway

${ }^{8}$ Department of Psychology, Faculty of Social and Educational Sciences, Norwegian University of Science and Technology (NTNU), Trondheim, Norway
}

entry/re-entry to work. Generalised estimated equations (GEE) regression analysis was used.

\section{Results}

Of 213 participants $80 \%$ were women. Mean age was 42 years. Main diagnoses of sickness certification were mental (38\%) and musculoskeletal (30\%) disorders.

The mean total number of days worked during the first year following the rehabilitation programme was marginally higher for participants in the intervention group (71 days) compared with the control group (68 days). Initially, the control group had higher entry/re-entry to work. After 6 months, the intervention group had surpassed the control group, and the proportion of participants working 1 day or more per week continued to increase. One year after discharge the intervention group had $87 \%$ increased odds (odds ratio 1.87, 95\% confidence interval 1.06-3.31, $p=0.031$ ), of entry/re-entry to competitive work $\geq 1$ day per week compared with the controls. Sensitivity analyses for the cut off 'minimum half-time work' and 'full-time work' showed similar patterns to the main analysis, but substantially increased odds were only seen for half-time work. The number needed to treat (NNT) was ten persons, meaning that an additional one in ten participants receiving boosted follow-up crossed the threshold from a nonparticipatory state to competitive work of 1 day or more per week. The mean cost per participant for the six-month intervention was 390.5 euros. There were on average six contacts per participant, and RTW coordinators spent in total seven hours per participant delivering follow-up.

\section{Conclusion and recommendations}

Participants receiving boosted RTW follow-up had substantially higher odds of having moved from non-participation to entering/re-entering paid work $\geq 1$ day when compared with the control group. Adding boosted follow-up by telephone after completing an occupational rehabilitation programme may augment the effect on RTW at limited cost.

Original publication: Hara, K.W., et al. Randomized Controlled Trial of Adding Telephone Follow-Up to an Occupational Rehabilitation Program to Increase Work Participation. J Occup Rehabil (2018) 28: 265. https://doi.org/10.1007/s10926-017-9711-4. 